\title{
Golay complementary array pairs
}

\author{
Jonathan Jedwab Matthew G. Parker
}

24 October 2006 (revised 28 April 2007)

\begin{abstract}
Constructions and nonexistence conditions for multi-dimensional Golay complementary array pairs are reviewed. A construction for a $d$-dimensional Golay array pair from a $(d+1)$-dimensional Golay array pair is given. This is used to explain and expand previously known constructive and nonexistence results in the binary case.
\end{abstract}

Keywords array, binary, complementary, complex-valued, construction, Golay, multidimensional, nonexistence, sequence

\section{Introduction}

We define a length s sequence to be a one-dimensional array $\mathcal{A}=(A[i])$ of complex-valued entries for which

$$
A[i]=0 \text { if } i<0 \text { or } i \geq s .
$$

Usually the sequence entries $\{A[i] \mid 0 \leq i<s\}$ are constrained to lie in a small finite set $S$ called the sequence alphabet. If $S=\left\{1, \xi, \xi^{2}, \ldots, \xi^{H-1}\right\}$ for some primitive $H$ th root of unity $\xi$ then $\mathcal{A}$ is an $H$-phase sequence. Particular cases of interest are the binary case $H=2$, for which $S=\{1,-1\}$, and the quaternary case $H=4$, for which $S=\{1, \sqrt{-1},-1,-\sqrt{-1}\}$. The ternary case $S=\{1,0,-1\}$ is not an $H$-phase sequence but has been much studied recently. The aperiodic autocorrelation function of a length $s$ sequence $\mathcal{A}=(A[i])$ is given by

$$
C_{\mathcal{A}}(u):=\sum_{i} A[i] \overline{A[i+u]} \text { for integer } u,
$$

where bar represents complex conjugation.

Since the 1950s, digital communications engineers have sought to identify binary sequences for which the absolute values of the aperiodic autocorrelation function are collectively small, for application in synchronisation, pulse compression and especially radar [27].

J. Jedwab is with Department of Mathematics, Simon Fraser University, 8888 University Drive, Burnaby BC, Canada V5A 1S6. He is grateful for support from NSERC of Canada.

M.G. Parker is with Department of Informatics, High Technology Center in Bergen, University of Bergen, Bergen 5020, Norway. He is grateful to Simon Fraser University for hospitality during 2006. 
¿From this point of view an ideal length $s$ binary sequence $\mathcal{A}$, known as a Barker sequence, is one for which

$$
\left|C_{\mathcal{A}}(u)\right| \leq 1 \text { for all } u \neq 0 .
$$

The only non-trivial lengths $s$ for which Barker sequences are known to exist are 2, 3, 4, 5, 7, 11 and 13, and it has been conjectured since at least 1963 [26] that no other sequence lengths are possible (see [18] for historical background and recent results).

Since it is apparent that the ideal behaviour given by a Barker sequence is unlikely to be achieved beyond length 13, researchers have explored several variations of the Barker condition for binary sequences. These include upper bounding the peak sidelobe level $\max _{u>0}\left|C_{\mathcal{A}}(u)\right|$ by an integer greater than 1 (see [20] for a summary of known results), or seeking to maximise the merit factor $s^{2} /\left(2 \sum_{u>0}\left[C_{\mathcal{A}}(u)\right]^{2}\right)$ (see [18] for a survey). A different approach, which we consider here, is to require that the sum of the aperiodic autocorrelation functions of a pair of length $s$ sequences $\mathcal{A}$ and $\mathcal{B}$ exhibits the ideal behaviour

$$
C_{\mathcal{A}}(u)+C_{\mathcal{B}}(u)=0 \text { for all } u \neq 0 .
$$

Such a pair of sequences is called a Golay complementary sequence pair (often abbreviated to Golay sequence pair) of length s, after Golay [14], [15]; Shapiro [24] independently studied the same object.

The initial investigation of Golay sequence pairs was restricted to the binary case. Binary Golay sequence pairs are known for lengths 2 and 10 [16], and 26 [17]. Infinitely many lengths of binary Golay sequence pairs can therefore be obtained from the following composition construction:

Theorem 1 (Turyn [28]). If there exist binary Golay sequence pairs of length $s_{1}$ and $s_{2}$ then there exists a binary Golay sequence pair of length $s_{1} s_{2}$.

Corollary 2. There exists a binary Golay sequence pair of length $2^{a} 10^{b} 26^{c}$ for all integer $a, b, c \geq 0$.

On the nonexistence side we have the following two results; the original proof of Theorem 4 in [9] was elegantly shortened in [10]:

Proposition 3 (Golay [16]). If there exists a binary Golay sequence pair of length $s>1$ then $s$ is even.

Proof. We give a short alternative proof to that in [16]. For a binary sequence $\mathcal{A}$ of length $s$, it is well-known (see, for example, [25]) that

$$
C_{\mathcal{A}}(u)+C_{\mathcal{A}}(u-s) \equiv s \quad(\bmod 4) \text { for all integer } u \text { satisfying } 0 \leq u<s
$$

Let $\mathcal{A}$ and $\mathcal{B}$ form a binary Golay sequence pair of length $s>1$. Add the congruence (1) for $\mathcal{A}$ to the congruence $(1)$ for $\mathcal{B}$, and set $u=1$ to obtain $0 \equiv 2 s \quad(\bmod 4)$.

Theorem 4 (Eliahou, Kervaire and Saffari [9], [10]). If there exists a binary Golay sequence pair of length s then s has no prime factor congruent to 3 modulo 4. 
Proposition 3 and Theorem 4 imply all known theoretical results on the nonexistence of binary Golay sequence pairs, including that $s$ must be the sum of two squares [16]. But the combination of Corollary 2, Proposition 3, and Theorem 4 still leaves infinitely many values of $s$ for which the existence of a binary Golay sequence pair of length $s$ is undecided. Borwein and Ferguson [1] extended previous exhaustive computer search results to show that all such undecided cases with $s<100$ give nonexistence.

Subsequently to Golay's work, several authors considered larger alphabets, including ternary [3], [5], [13]; quaternary [4]; $2^{h}$-phase [6]; $H$-phase for even $H$ [23]; and the case $|A[i]|=1$ for $0 \leq i<s[2]$. Fiedler, Jedwab and Parker [11] recently gave a framework of constructions for $H$-phase sequences for even $H$, from which all previously known $2^{h}$-phase Golay sequence pairs of length $2^{m}$ can be obtained in explicit algebraic normal form.

We have described one approach to the apparent scarcity of Barker sequences, namely to replace a single sequence by a pair of sequences. An alternative approach is to generalise $\mathcal{A}$ from one dimension to multiple dimensions in the hope of a richer existence pattern. We define an $s_{1} \times \cdots \times s_{r}$ array to be an $r$-dimensional array $\mathcal{A}=\left(A\left[i_{1}, \ldots, i_{r}\right]\right)$ of complex-valued entries for which

$$
A\left[i_{1}, \ldots, i_{r}\right]=0 \text { if, for any } k \in\{1,2, \ldots, r\}, i_{k}<0 \text { or } i_{k} \geq s_{k} .
$$

Binary, ternary, quaternary and other special cases of arrays are defined as for sequences. The aperiodic autocorrelation function of an $s_{1} \times \cdots \times s_{r}$ array $\mathcal{A}=\left(A\left[i_{1}, \ldots, i_{r}\right]\right)$ is given by

$$
C_{\mathcal{A}}\left(u_{1}, \ldots, u_{r}\right):=\sum_{i_{1}} \ldots \sum_{i_{r}} A\left[i_{1}, \ldots, i_{r}\right] \overline{A\left[i_{1}+u_{1}, \ldots, i_{r}+u_{r}\right]} \text { for integer } u_{1}, \ldots, u_{r} .
$$

By analogy with the one-dimensional case, an $s_{1} \times \cdots \times s_{r}$ Barker array is defined to be an $s_{1} \times \cdots \times s_{r}$ binary array for which

$$
\left|C_{\mathcal{A}}\left(u_{1}, \ldots, u_{r}\right)\right| \leq 1 \text { for all }\left(u_{1}, \ldots, u_{r}\right) \neq(0, \ldots, 0) .
$$

The array $\left[\begin{array}{rr}1 & 1 \\ 1 & -1\end{array}\right]$ is a $2 \times 2$ Barker array, but there are no other possible sizes for an $r$-dimensional Barker array with $r>1$ :

Theorem 5 (Davis, Jedwab and Smith [7]). There are no $s_{1} \times s_{2}$ Barker arrays having $s_{1}, s_{2}>1$ except when $s_{1}=s_{2}=2$.

Theorem 6 (Jedwab and Parker [19]). There are no $s_{1} \times \cdots \times s_{r}$ Barker arrays having $r>2$ and each $s_{k}>1$.

We have now described two variations of a Barker sequence: a Golay sequence pair, and a Barker array. The first variation produces an infinite family of binary sequence pairs with length of the form $2^{a} 10^{b} 26^{c}$, while the second produces no new binary examples except for size $2 \times 2$. It is natural to combine these two variations by seeking a Golay 
array pair. Accordingly, we define an $s_{1} \times \cdots \times s_{r}$ Golay (complementary) array pair to be a pair of $s_{1} \times \cdots \times s_{r}$ arrays $\mathcal{A}$ and $\mathcal{B}$ for which

$$
C_{\mathcal{A}}\left(u_{1}, \ldots, u_{r}\right)+C_{\mathcal{B}}\left(u_{1}, \ldots, u_{r}\right)=0 \text { for all }\left(u_{1}, \ldots, u_{r}\right) \neq(0, \ldots, 0) .
$$

A central question is for what sizes $s_{1} \times \cdots \times s_{r}$ such a pair exists. A further question, considered in [12], is how many different Golay array pairs of a given size exist.

In 1978 Ohyama, Honda and Tsujiuchi [22] proposed the use of a two-dimensional binary Golay array pair for X-ray and gamma ray coded aperture imaging. They gave a construction to increase the size of such a pair from $s_{1} \times s_{2}$ to $2 s_{1} \times s_{2}$, and by recursive application to a $2 \times 1$ starting pair constructed a $2^{a} \times 2^{b}$ binary Golay array pair for all integer $a, b \geq 0$.

In 1985 Lüke [21] indicated (without proof) a generalisation of Theorem 1 to multidimensional arrays, whose recursive application leads to:

Theorem 7 (Lüke [21]). If there exist binary Golay sequence pairs of length $s_{1}, \ldots, s_{r}$ then there exists an $s_{1} \times \cdots \times s_{r}$ binary Golay array pair.

By Corollary 2 we deduce:

Corollary 8. There exists an $s_{1} \times \cdots \times s_{r}$ binary Golay array pair, where each $s_{k}$ takes the form $2^{a_{k}} 10^{b_{k}} 26^{c_{k}}$ for integer $a_{k}, b_{k}, c_{k} \geq 0$.

For an $s_{1} \times \cdots \times s_{r}$ array $\mathcal{A}=\left(A\left[i_{1}, \ldots, i_{r}\right]\right)$, write $\mathcal{A}^{*}:=\overline{\left(A\left[s_{1}-1-i_{1}, \ldots, s_{r}-1-i_{r}\right]\right)}$ In 1992 Dymond [8] conducted a detailed study of (mostly binary) Golay array pairs, showing that Golay's concatentation, interleaving, and composition constructions for binary Golay sequence pairs [16] can all be generalised to multiple dimensions. She also proved an important generalisation of Theorem 1 to the construction of multi-dimensional arrays:

Theorem 9 (Dymond [8, Theorem 4.24]). Let $\otimes$ represent the Kronecker product of arrays. Suppose that $\mathcal{A}$ and $\mathcal{B}$ form an $s_{1} \times \cdots \times s_{r}$ binary Golay array pair, and that $\mathcal{C}$ and $\mathcal{D}$ form a $t_{1} \times \cdots \times t_{r}$ binary Golay array pair (where any of the $s_{k}$ and $t_{k}$ can take the value 1$)$. Then the arrays

$$
\begin{aligned}
& \mathcal{A} \otimes\left(\frac{\mathcal{C}+\mathcal{D}}{2}\right)+\mathcal{B} \otimes\left(\frac{\mathcal{C}-\mathcal{D}}{2}\right), \\
& \mathcal{A} \otimes\left(\frac{\mathcal{C}^{*}-\mathcal{D}^{*}}{2}\right)-\mathcal{B} \otimes\left(\frac{\mathcal{C}^{*}+\mathcal{D}^{*}}{2}\right)
\end{aligned}
$$

form an $s_{1} t_{1} \times \cdots \times s_{r} t_{r}$ binary Golay array pair.

Using Theorem 9 we can construct a binary Golay array pair of size $s_{1} \times \cdots \times s_{r-1} \times s_{r}$ from binary array Golay pairs of size $s_{1} \times \cdots \times s_{r-1} \times 1$ and $1 \times \cdots \times 1 \times s_{r}$, from which Theorem 7 can be recovered by repeated application. But although Theorem 9 is a more general construction than Theorem 7, it does not produce new sizes of binary Golay array pairs beyond those given in Corollary 8 . 
On the nonexistence side, Dymond [8] proved that if an $s_{1} \times \cdots \times s_{r}$ binary Golay array pair exists then $\prod_{i=1}^{r} s_{i}$ is an even sum of two squares (generalising Golay's results for the binary sequence pair case [16]). She ruled out the existence of a binary Golay array pair of size $2 \times 5$ by exhaustive search, and could find no examples of size $3 \times 6,2 \times 9$ or $2 \times 3 \times 3$ by extensive (though non-exhaustive) search [8, p. 143].

We will use our main result, Theorem 11, to explain many of these previously known constructive and nonexistence results.

\section{Reduction of the dimension of a Golay array pair}

In [19] the authors observed that a $(d+1)$-dimensional binary array can be mapped to a $d$-dimensional binary array so that the aperiodic autocorrelation functions of both arrays are closely related. The proof given in [19] does not make use of the condition that the array is binary, and holds without modification for arrays of complex-valued entries:

Lemma 10 (Jedwab and Parker [19, Lemma 2.1]). For integer $r \geq 0$, let $\mathcal{A}=$ $\left(A\left[i, j, i_{1}, \ldots, i_{r}\right]\right)$ be an $s \times t \times s_{1} \times \cdots \times s_{r}$ array. Define the $s t \times s_{1} \times \cdots \times s_{r}$ array $\psi(\mathcal{A})=\left(B\left[m, i_{1}, \ldots, i_{r}\right]\right)$ by

$B\left[t i+j, i_{1}, \ldots, i_{r}\right]:=A\left[i, j, i_{1}, \ldots, i_{r}\right]$ for $0 \leq i<s, 0 \leq j<t, 0 \leq i_{k}<s_{k}(k=1, \ldots, r)$.

Then, for all integer $u, v, u_{1}, \ldots, u_{r}$, where $0 \leq v<t$,

$$
C_{\psi(\mathcal{A})}\left(t u+v, u_{1}, \ldots, u_{r}\right)=C_{\mathcal{A}}\left(u, v, u_{1}, \ldots, u_{r}\right)+C_{\mathcal{A}}\left(u+1, v-t, u_{1}, \ldots, u_{r}\right) .
$$

Lemma 10 expresses each aperiodic autocorrelation $C_{\psi(\mathcal{A})}$ as the sum of exactly two terms $C_{\mathcal{A}}$ (one or both of which might be trivially zero, according to the values of $u$ and $v$ ). We now use this result to show that the existence of a $(d+1)$-dimensional Golay array pair implies the existence of a $d$-dimensional Golay array pair.

Theorem 11. For integer $r \geq 0$, suppose that $\mathcal{A}$ and $\mathcal{B}$ form an $s \times t \times s_{1} \times \cdots \times s_{r}$ Golay array pair over an alphabet $S$. Then $\psi(\mathcal{A})$ and $\psi(\mathcal{B})$, as defined in Lemma 10 , form an st $\times s_{1} \times \cdots \times s_{r}$ Golay array pair over $S$.

Proof. Fix integers $u, v, u_{1}, \ldots, u_{r}$, where $0 \leq v<t$ and $\left(u, v, u_{1}, \ldots, u_{r}\right) \neq(0, \ldots, 0)$. By Lemma 10,

$$
\begin{aligned}
C_{\psi(\mathcal{A})}\left(t u+v, u_{1}, \ldots, u_{r}\right)+C_{\psi(\mathcal{B})}\left(t u+v, u_{1}, \ldots, u_{r}\right) \\
=C_{\mathcal{A}}\left(u, v, u_{1}, \ldots, u_{r}\right)+C_{\mathcal{A}}\left(u+1, v-t, u_{1}, \ldots, u_{r}\right)+ \\
\quad C_{\mathcal{B}}\left(u, v, u_{1}, \ldots, u_{r}\right)+C_{\mathcal{B}}\left(u+1, v-t, u_{1}, \ldots, u_{r}\right) \\
=0
\end{aligned}
$$

since $\mathcal{A}$ and $\mathcal{B}$ form a Golay array pair. Therefore $\psi(\mathcal{A})$ and $\psi(\mathcal{B})$ form an $s t \times s_{1} \times \cdots \times s_{r}$ Golay array pair, and are defined over the same alphabet as $\mathcal{A}$ and $\mathcal{B}$.

Repeated application of Theorem 11 gives: 
Corollary 12. If there exists an $s_{1} \times \cdots \times s_{r}$ Golay array pair then there exists a Golay sequence pair of length $\prod_{k=1}^{r} s_{k}$ over the same alphabet.

Theorem 11 also allows an alternative formulation of Theorem 9 that is conceptually simpler: specialise Theorem 9 to the case where $\mathcal{A}$ and $\mathcal{B}$ have size $s_{1} \times \cdots \times s_{r} \times 1 \times$ $\cdots \times 1$ (with $v 1$ 's), and $\mathcal{C}$ and $\mathcal{D}$ have size $1 \times \cdots \times 1 \times t_{1} \times \cdots \times t_{v}$ (with $r 1$ 's). The Kronecker product then simplifies to the tensor product, giving a constructed array of size $s_{1} \times \cdots \times s_{r} \times t_{1} \times \cdots \times t_{v}$. We can recover the original form of Theorem 9 from this simpler formulation by setting $v=r$ and then applying Theorem 11 .

\section{Application to the binary case}

Theorem 11 and Corollary 12 are of particular interest in the binary case, where we have:

Proposition 13. Up to reordering of dimensions, an $s_{1} \times \cdots \times s_{r}$ binary Golay array pair with $1<\prod_{k=1}^{r} s_{k}<100$ exists for precisely the following sizes, together with the derived sizes arising from Theorem 11:

$2, \quad 2 \times 2, \quad 2 \times 2 \times 2, \quad 2 \times 2 \times 2 \times 2, \quad 2 \times 2 \times 2 \times 2 \times 2, \quad 2 \times 2 \times 2 \times 2 \times 2 \times 2$,

$10,2 \times 10, \quad 2 \times 2 \times 10,2 \times 2 \times 2 \times 10$,

$26, \quad 2 \times 26$.

Proof. All the listed sizes of binary Golay array pairs exist, by Corollary 8 . We now show that, up to reordering of dimensions, no other sizes satisfying the stated conditions exist.

Suppose there exists an $s_{1} \times \cdots \times s_{r}$ binary Golay array pair with $1<\prod_{k=1}^{r} s_{k}<100$. By Corollary 12, a binary Golay sequence pair of length $\prod_{k=1}^{r} s_{k}$ exists. The computer search results of [1] then force

$$
\prod_{k=1}^{r} s_{k} \in\{2,4,8,16,32,64,10,20,40,80,26,52\} .
$$

By Theorem 11, it is now sufficient to rule out the existence of binary Golay array pairs of the following sizes:

$$
2 \times 5, \quad 4 \times 5, \quad 8 \times 5, \quad 16 \times 5, \quad 2 \times 13, \quad 4 \times 13
$$

(since, for example, the nonexistence of a binary Golay array pair of size $16 \times 5$ implies the nonexistence of binary Golay array pairs of size $2 \times 2 \times 2 \times 2 \times 5,2 \times 2 \times 4 \times 5,2 \times 8 \times 5$, and $4 \times 4 \times 5$ ). Since there exists a binary Golay sequence pair of length 2 , by Theorem 9 it is then sufficient to rule out the existence of binary Golay array pairs of just two sizes:

$$
16 \times 5 \text { and } 4 \times 13 \text {. }
$$

We describe an efficient computer procedure by which this ruling out can be done for size $16 \times 5$; the procedure for size $4 \times 13$ is similar. If $\mathcal{A}$ and $\mathcal{B}$ form a binary Golay array pair of size $16 \times 5$ then, by Theorem $11, \psi(\mathcal{A})$ and $\psi(\mathcal{B})$ form a binary Golay sequence 
pair of length 80 (where the mapping $\psi$ is defined in Lemma 10). Now the exact number and structure of binary Golay sequence pairs of length 80 is known from [1], so we can apply the inverse map $\psi^{-1}$ to all such sequence pairs and test the resulting array pairs of size $16 \times 5$ for the Golay property. Since we find that no such array pair is a Golay array pair, we conclude that there is no $16 \times 5$ binary Golay array pair.

In particular, Proposition 13 confirms Dymond's computer search results [8] that established the nonexistence of a binary Golay array pair of size $2 \times 5$ and suggested the nonexistence of binary Golay array pairs of size $3 \times 6,2 \times 9$ and $2 \times 3 \times 3$ (see Section 1).

The proof of Proposition 13 demonstrates that the fundamental object of interest is a Golay array pair having the largest possible number of dimensions, and that other Golay array pairs arising from Theorem 11 should be regarded as derived objects. For example, the existence of a $2 \times 2 \times 10$ binary Golay array pair given by Corollary 8 implies the existence of binary Golay array pairs of size $2 \times 20,4 \times 10$, and 40 . Similarly, if the nonexistence of a binary Golay array pair of size $4 \times 25$ were established, it would imply the nonexistence of binary Golay array pairs of size $2 \times 2 \times 25,4 \times 5 \times 5$, and $2 \times 2 \times 5 \times 5$.

The following two nonexistence results generalise Proposition 3 and Theorem 4 from binary sequences to multi-dimensional binary arrays. Both results could be established by adapting the original proofs to multiple dimensions, but follow directly as a consequence of Corollary 12:

Corollary 14. If there exists an $s_{1} \times \cdots \times s_{r}$ binary Golay array pair then $\prod_{k=1}^{r} s_{k}=1$ or $\prod_{k=1}^{r} s_{k}$ is even.

Proof. Combine Proposition 3 and Corollary 12.

Corollary 15. If there exists an $s_{1} \times \cdots \times s_{r}$ binary Golay array pair then no $s_{k}$ has a prime factor congruent to 3 modulo 4.

Proof. Combine Theorem 4 and Corollary 12.

Corollary 14 was previously established by Dymond [8]. If an $s_{1} \times \cdots \times s_{r}$ binary Golay array pair exists then Corollary 15 implies that $\prod_{k=1}^{r} s_{k}$ is the sum of two squares, as was also previously established by Dymond [8].

\section{Conclusion}

We conclude by summarising the results of the paper, and presenting some open questions.

Historically, the primary theoretical and practical motivation for the generalisation from Golay sequence pairs to Golay array pairs was to enlarge the set of available objects. Comparison of Corollaries 2 and 8 demonstrates some success for this approach in the binary case, and prompts the question:

1. Does the existence of an $s_{1} \times \cdots \times s_{r}$ binary Golay array pair imply the existence of a binary Golay sequence pair of length $s_{k}$ for each $k$ ? 
A subsidiary question suggested by the proof of Proposition 13 is:

2. Does the existence of an $s_{1} \times \cdots \times s_{r}$ binary Golay array pair with each $s_{k}>1$ imply that each $s_{k}$ is even?

Golay array pairs are not just a possible source of further examples: we have shown that a Golay array pair having the largest possible number of dimensions is in fact a fundamental object of interest, from which lower-dimensional Golay array pairs can be derived via Theorem 11.

In the quaternary case, Craigen, Holzmann and Kharaghani [4] showed by computer search that a length $s$ Golay sequence pair exists for $1<s \leq 22$ precisely when

$$
s \in\{2,3,4,5,6,8,10,11,12,13,16,18,20,22\} .
$$

We ask:

3. Does an array viewpoint shed light on the existence pattern for quaternary Golay sequence pairs, and in particular the conjectures in [4]?

The focus of this paper is the set of sizes for which a Golay array pair can exist, especially in the binary case. A further paper [12] considers the explicit construction, structure, and enumeration of Golay array pairs of a given size $s_{1} \times \cdots \times s_{r}$, especially when $\prod_{k=1}^{r} s_{k}$ is a power of 2 .

\section{References}

[1] P.B. Borwein and R.A. Ferguson. A complete description of Golay pairs for lengths up to 100. Mathematics of Computation, 73:967-985, 2003.

[2] S.Z. Budišin. New complementary pairs of sequences. Electron. Lett., 26:881-883, 1990.

[3] R. Craigen, S. Georgiou, W. Gibson, and C. Koukouvinos. Further explorations into ternary complementary pairs. J. Combin. Theory (Series A), 113:952-965, 2006.

[4] R. Craigen, W. Holzmann, and H. Kharaghani. Complex Golay sequences: structure and applications. Discrete Math., 252:73-89, 2002.

[5] R. Craigen and C. Koukouvinos. A theory of ternary complementary pairs. J. Combin. Theory (Series A), 96:358-375, 2001.

[6] J.A. Davis and J. Jedwab. Peak-to-mean power control in OFDM, Golay complementary sequences, and Reed-Muller codes. IEEE Trans. Inform. Theory, 45:2397-2417, 1999.

[7] J.A. Davis, J. Jedwab, and K.W. Smith. Proof of the Barker array conjecture. Proc. Amer. Math. Soc., 135:2011-2018, 2007. 
[8] M. Dymond. Barker arrays: existence, generalization and alternatives. $\mathrm{PhD}$ thesis, University of London, 1992.

[9] S. Eliahou, M. Kervaire, and B. Saffari. A new restriction on the lengths of Golay complementary sequences. J. Combin. Theory (A), 55:49-59, 1990.

[10] S. Eliahou, M. Kervaire, and B. Saffari. On Golay polynomial pairs. Advances App. Math., 12:235-292, 1991.

[11] F. Fiedler, J. Jedwab, and M.G. Parker. A framework for the construction of Golay sequences. IEEE Trans. Inform. Theory, 2006. Submitted.

[12] F. Fiedler, J. Jedwab, and M.G. Parker. A multi-dimensional approach to the construction and enumeration of Golay complementary sequences. J. Combin. Theory (A), 2006. Submitted.

[13] A. Gavish and A. Lempel. On ternary complementary sequences. IEEE Trans. Inform. Theory., 40:522-526, 1994.

[14] M.J.E. Golay. Multislit spectroscopy. J. Opt. Soc. Amer., 39:437-444, 1949.

[15] M.J.E. Golay. Static multislit spectrometry and its application to the panoramic display of infrared spectra. J. Opt. Soc. Amer., 41:468-472, 1951.

[16] M.J.E. Golay. Complementary series. IRE Trans. Inform. Theory, IT-7:82-87, 1961.

[17] M.J.E. Golay. Note on "Complementary series". Proc. IRE, 50:84, 1962.

[18] J. Jedwab. A survey of the merit factor problem for binary sequences. In T. Helleseth et al., editors, Sequences and Their Applications - Proceedings of SETA 2004, volume 3486 of Lecture Notes in Computer Science, pages 30-55. Springer-Verlag, Berlin Heidelberg, 2005.

[19] J. Jedwab and M.G. Parker. There are no Barker arrays having more than two dimensions. Designs, Codes and Cryptography, 2007. To appear.

[20] J. Jedwab and K. Yoshida. The peak sidelobe level of families of binary sequences. IEEE Trans. Inform. Theory, 52:2247-2254, 2006.

[21] H.D. Lüke. Sets of one and higher dimensional Welti codes and complementary codes. IEEE Trans. Aerospace Electron. Systems, AES-21:170-179, 1985.

[22] N. Ohyama, T. Honda, and J. Tsujiuchi. An advanced coded imaging without side lobes. Optics Comm., 27:339-344, 1978.

[23] K.G. Paterson. Generalized Reed-Muller codes and power control in OFDM modulation. IEEE Trans. Inform. Theory, 46:104-120, 2000.

[24] H.S. Shapiro. Extremal problems for polynomials and power series. Master's thesis, Mass. Inst. of Technology, 1951. 
[25] R. Turyn and J. Storer. On binary sequences. Proc. Amer. Math. Soc., 12:394-399, 1961.

[26] R.J. Turyn. On Barker codes of even length. Proceedings of the IEEE, 51:1256, 1963.

[27] R.J. Turyn. Sequences with small correlation. In H.B. Mann, editor, Error Correcting Codes, pages 195-228. Wiley, New York, 1968.

[28] R.J. Turyn. Hadamard matrices, Baumert-Hall units, four-symbol sequences, pulse compression, and surface wave encodings. J. Combin. Theory (A), 16:313-333, 1974. 